\title{
Characterization of Jasmonoyl-Isoleucine (JA-lle) Hormonal Catabolic Pathways in Rice upon Wounding and Salt Stress
}

\author{
Mohamed Hazman ${ }^{1,2}$, Martin Sühnel ${ }^{3}$, Sandra Schäfer ${ }^{3}$, Julie Zumsteg ${ }^{1}$, Agnès Lesot ${ }^{1}$, Fréderic Beltran ${ }^{4}$, \\ Valentin Marquis ${ }^{1}$, Laurence Herrgott ${ }^{1}$, Laurence Miesch ${ }^{4}$, Michael Riemann ${ }^{3^{*+}}$ and Thierry Heitz ${ }^{1{ }^{*+}}$ (D)
}

\begin{abstract}
Background: Jasmonate (JA) signaling and functions have been established in rice development and response to a range of biotic or abiotic stress conditions. However, information on the molecular actors and mechanisms underlying turnover of the bioactive jasmonoyl-isoleucine (JA-lle) is very limited in this plant species.

Results: Here we explored two gene families in rice in which some members were described previously in Arabidopsis to encode enzymes metabolizing JA-lle hormone, namely cytochrome P450 of the CYP94 subfamily (CYP94, 20 members) and amidohydrolases (AH, 9 members). The CYP94D subclade, of unknown function, was most represented in the rice genome with about 10 genes. We used phylogeny and gene expression analysis to narrow the study to candidate members that could mediate JA-lle catabolism upon leaf wounding used as mimic of insect chewing or seedling exposure to salt, two stresses triggering jasmonate metabolism and signaling. Both treatments induced specific transcriptional changes, along with accumulation of JA-lle and a complex array of oxidized jasmonate catabolites, with some of these responses being abolished in the JASMONATE RESISTANT 1 (jar1) mutant. However, upon response to salt, a lower dependence on JAR1 was evidenced. Dynamics of CYP94B5, CYP94C2, CYP94C4 and AH7 transcripts matched best the accumulation of JA-lle catabolites. To gain direct insight into JA-lle metabolizing activities, recombinant expression of some selected genes was undertaken in yeast and bacteria. CYP94B5 was demonstrated to catalyze C12-hydroxylation of JA-lle, whereas similarly to its Arabidopsis bi-functional homolog IAR3, AH8 performed cleavage of JA-lle and auxinalanine conjugates.
\end{abstract}

Conclusions: Our data shed light on two rice gene families encoding enzymes related to hormone homeostasis. Expression data along with JA profiling and functional analysis identifies likely actors of JA-lle catabolism in rice seedlings. This knowledge will now enable to better understand the metabolic fate of JA-lle and engineer optimized JA signaling under stress conditions.

Keywords: Jasmonate catabolism, Wounding, Salt stress, JA-lle, CYP94, Amidohydrolase

\section{Background}

Jasmonate hormones (JAs) play major roles in plant development and their responses to environmental challenges. Essential functions of this hormonal pathway have been demonstrated in vegetative or reproductive processes, and adaptation to an array of adverse conditions, particularly in

\footnotetext{
* Correspondence: michael.riemann@kit.edu; thierry.heitz@ibmp-cnrs.unistra.fr ${ }^{\dagger}$ Michael Riemann and Thierry Heitz contributed equally to this work.

${ }^{3}$ Karlsruhe Institute of Technology, Botanical Institute, Karlsruhe, Germany 'Institut de Biologie Moléculaire des Plantes (IBMP) du CNRS, Université de Strasbourg, Strasbourg, France

Full list of author information is available at the end of the article
}

Arabidopsis and other dicotyledonous plants (Wasternack and Hause 2013). Through complex interplays with other phytohormones, JAs control a wide spectrum of induced responses, including the accumulation of defense proteins, or enzymes directing the formation of specialized metabolites, both increasing survival capacity against herbivorous insects or necrotrophic microbial pathogens (Campos et al. 2014). In addition, JA signaling also impacts tolerance to different types of abiotic stress (Kazan 2015), making this hormone a critical hub for translating environmental cues into relevant physiological adaptations. 
The core jasmonate biosynthetic pathway is initiated when linolenic acid from plastidial membranes is converted by the successive action of lipoxygenase, allene oxide synthase and allene oxide cyclase into the precursor 12-oxophytodienoic acid (OPDA). This compound is then further reduced by OPDA-reductase 3 (OPR3) followed by three rounds of $\beta$-oxidation to form the inactive pro-hormone jasmonic acid (JA) (Schaller and Stintzi 2009). Among many possible modification routes described for JA (Wasternack and Hause 2013), its conjugation to isoleucine by Jasmonate Resistant 1 (JAR1) constitutes the critical activation step, and resulting jasmonoyl-isoleucine (JA-Ile) acts as the master regulator of most JA responses in higher plants (Campos et al. 2014; Heitz et al. 2016). This occurs when JA-Ile promotes assembly of the F-box protein CORONATINE INSENSITIVE 1 (COI1) with one of numerous JASMONATE-ZIM-domain (JAZ) repressor proteins and disrupts their transcription-repressing function at promoter regions of target genes. Upon ligand-dependent co-receptor assembly, JAZ are tagged by ubiquitin E3 ligase complexes before proteolytic degradation, allowing transcription of JAresponsive genes to proceed and execute the numerous adaptation responses (Chini et al. 2007; De Geyter et al. 2012; Thines et al. 2007).

In this mechanism, the levels and dynamics of JA-Ile are of crucial importance to direct a timely orchestration of transcriptional cascades ( $\mathrm{Du}$ et al. 2017; Hickman et al. 2017). Many biotic and abiotic stress conditions induce accumulation of JA/JA-Ile and presumed catabolic derivatives and only recently have new metabolic relationships been established within this hormone family (Heitz et al. 2016; Koo 2018). In particular, metabolic studies in Arabidopsis have revealed the existence of two enzymatic JA-Ile turnover routes that are induced along with biosynthetic and signaling pathways (Additional file 1: Figure S1). The first one consists in a two-step oxidation mediated by three cytochromes $\mathrm{P} 450$ of the 94 family (CYP94B1, B3 and C1) and the resulting $12 \mathrm{OH}-\mathrm{JA}-\mathrm{Ile}$ and $12 \mathrm{COOH}-\mathrm{JA}-\mathrm{Ile}$ products have strongly reduced capacity to promote COI1-JAZ co-receptor formation (Aubert et al. 2015; Heitz et al. 2012; Kitaoka et al. 2011; Koo et al. 2011, 2014). They are therefore considered as largely inactive derivatives. The second JA-Ile elimination pathway consists in conjugate cleavage by IAR3 and ILL6, two members of a multi-functional amido-hydrolase family, that release JA from JA-Ile, but also 12OH-JA from 12OH-JA-Ile (Widemann et al. 2013; Woldemariam et al. 2012; Zhang et al. 2016). Loss and mostly gain-of-function experiments have established that these enzymes deplete specifically JA-Ile hormone pools to attenuate JA signaling and contribute to the proper regulation and termination of JA-mediated processes. Despite of their importance, the nature and regulation of JA-Ile turnover pathways have not been investigated in crop species and may have significant impact on some agricultural traits with regards to stress tolerance. Rice is particularly suited for such a purpose as a model cereal and a major food crop. Recent research has identified candidate genes for most components of JA biosynthetic and signaling pathway and several features have been addressed functionally (Dhakarey et al. 2016; Liu et al. 2015; Riemann et al. 2015). Functions were described for example in photomorphogenesis (Svyatyna et al. 2014), spikelet development (Cai et al. 2014; Liu et al. 2017), control in the transition between juvenile and adult phases (Hibara et al. 2016), defense against insects (Lu et al. 2015; Ye et al. 2012) and pathogens (Riemann et al. 2013; Yamada et al. 2012). In addition, significant impacts of the JA pathway on abiotic stress tolerance were reported in this species (Dhakarey et al. 2016). The most documented case is probably salt stress resilience for which JA signaling seems to be detrimental, based on several reports. We showed previously that a JA-deficient rice mutant performs better upon salt exposure than wild-type (Hazman et al. 2015)

Similarly, suppressing (OsJAZ9) or enhancing (OsJAZ8) salt-induction of JAZ repressors improves tolerance to this stress (Peethambaran et al. 2018; Wu et al. 2015). This data supports a previous report by Kurotani and co-workers (Kurotani et al. 2015a) showing that a rice line overexpressing CYP94C2b, a putative ortholog of the JA-Ile-oxidase CYP94C1 in Arabidopsis displays enhanced survival upon salt exposure. This notion was further extended by the finding of a positive correlation between expression levels of this gene and salt tolerance in an extensive collection of tolerant rice lines (Kurotani et al. 2015b), suggesting a possible link between JA-Ile catabolism and salt stress tolerance.

In the present work, we explored rice JA-Ile catabolism under two distinct stresses known to trigger JA biosynthesis and signaling, namely mechanical wounding used as a proxy for attack by chewing insects, and salt exposure as a relevant abiotic stress that increasingly threatens rice productivity under agricultural conditions (Shrivastava and Kumar 2015). We established detailed kinetic jasmonate profiles in seedlings, including catabolic derivatives, and examined the requirement of the conjugating enzyme JAR1 for each stress. We next mined the rice genome to explore the two known gene families, CYP94 and amido-hydrolase $(A H)$, in which some Arabidopsis members encode JA-Ilemetabolizing activities as described above. We identified through phylogenic analysis and gene expression studies candidate rice members that could potentially encode such activities. By recombinant protein expression, we were able to demonstrate for one CYP94 and one AH JA-Ile oxidation or cleaving activities, respectively. The study sheds light onto new players of JA 
hormone turnover and increases our understanding of JA-Ile homeostasis in rice.

\section{Results}

Occurrence and Diversity of CYP94 and Amidohydrolase $(A H)$ Genes in the Rice Genome

The rice complement of JA-Ile catabolic genes/enzymes was explored, based on similarity search using known Arabidopsis sequences. CYP94 gene family was reported previously to contain about 18 distinct genes in the sequenced genome of Nipponbare rice (Widemann et al. 2015). Here we report 20 OsCYP94-related loci from the MSU database, for which not all have counterparts in the RAP database. OsCYP94 predicted proteins display a particular subclade enrichment. Like Arabidopsis, rice bears no subclade A member (Fig. 1a) and exhibits only 2 subclade B proteins, OsCYP94B4 and OsCYP94B5 along with 4 potential members in subclade $\mathrm{C}$,
OsCYP94C2a, OsCYP94C2b, OsCYP94C3 and OsCYP94C 4. In these latter two subclades, JA-Ile oxidases have been characterized in Arabidopsis (Heitz et al. 2012; Kitaoka et al. 2011; Koo et al. 2011). Subclade D and a new grass-specific subclade $E$, both of unknown function, are particularly diversified in rice with 11 and 3 members, respectively. These data indicate considerable CYP94 expansion and diversification in the rice genome.

Rice amidohydrolase (AH) gene family was similarly investigated and 9 predicted proteins were identified. Figure $1 \mathrm{~b}$ shows their phylogenetic relationships with the 7 Arabidopsis AH protein sequences (Rampey et al. 2004). Five members (OsAH2 through OsAH6) cluster with AtILR1 that prefers auxin-amino acid conjugates as substrates (Zhang et al. 2016). Two other isoforms, OsAH1 and OsAH9 were more distantly related to previously characterized enzymes. The closest rice homolog of AtIAR3,

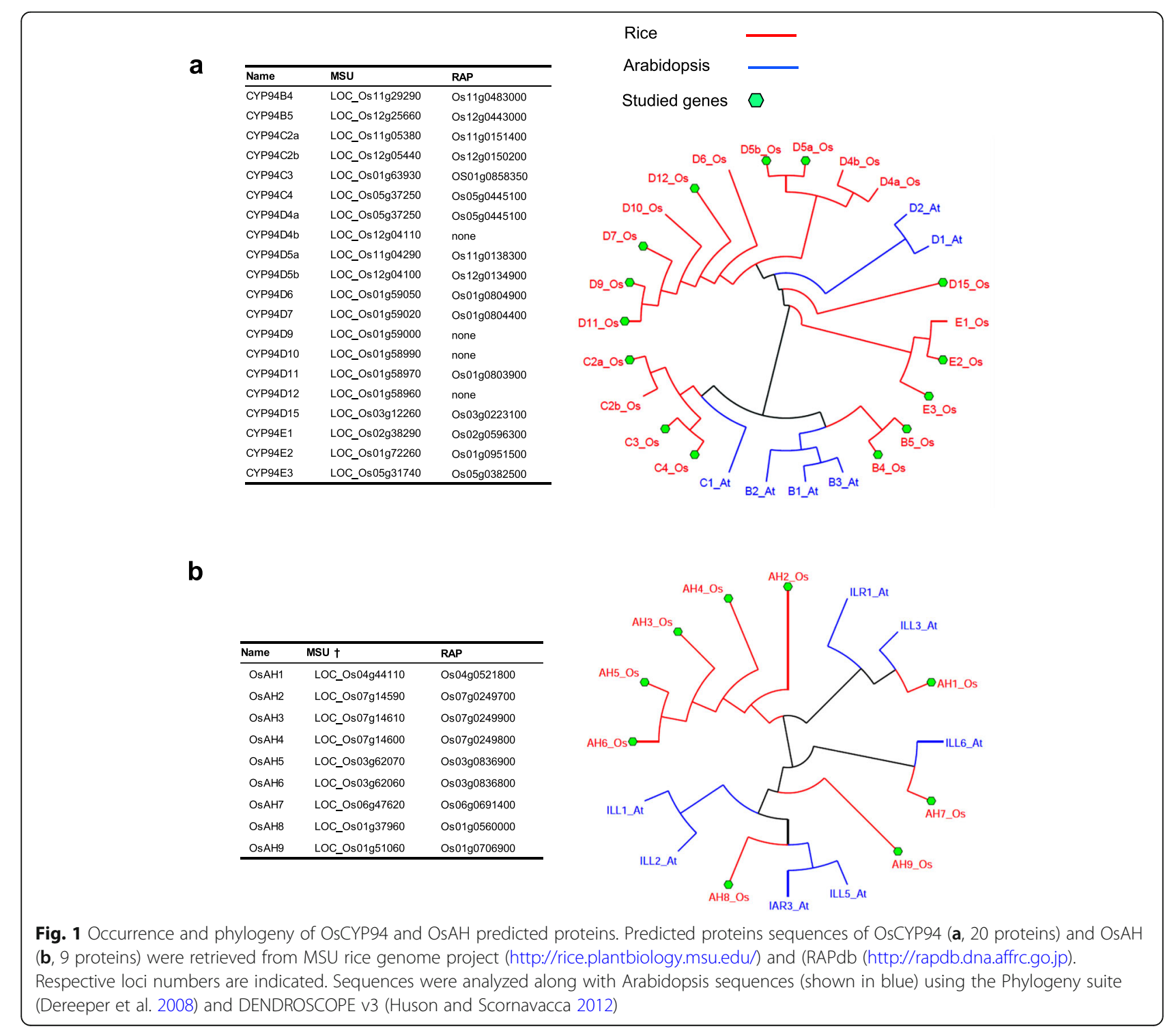


the conserved bifunctional AH cleaving both JA-amino acid and indole acetic acid-amino acid conjugates (Widemann et al. 2013; Zhang et al. 2016) was OsAH8 (78/64\% similar/identical). Finally, OsAH7 protein sequence was closely related $(75 / 63 \%$ similar/identical) to JA-Ile-specific AtILL6.

\section{Specific CYP94 and AH Gene Isoforms Display Dynamic Expression upon Wound Stress}

To investigate stress responsiveness of members in both gene families, expression studies were conducted in leaves using kinetic responses to wounding. Specific primer pairs were designed for genes with unique 3'UTR sequences available from databases, comprising 14 OsCYP94 genes and all 9 OsAH genes (Fig. 1). This criterion was met for CYP94C2a, but not for the very similar (91\%) and formerly reported CYP94C2b gene (Kurotani et al. 2015a) for which no specific primer pair could be designed. Most analyzed OsCYP94 genes exhibited wound-induced expression, either as a rapid and transient pulse peaking at $0.5 \mathrm{~h}$ postwounding (hpw), as for CYP94B4, CYP94B5, CYP94C2a, CYP94C3, CYP94C4, or as a steady increase over the $6 \mathrm{~h}$ period studied, for CYP94D5a, CYP94D7, CYP94D9, CYP 94E2 (Additional file 2: Figure S2). However, overall expression levels were extremely variable between genes, and ranged over 3 orders of magnitude. Despite of detectable wound-triggered changes, some isoforms displayed very low expression levels, like CYP4B4, CYP94C3, CYP94D5, C YP94D11, CYP94D12, CYP94D15, and CYP94E2, and were not further studied.

A similar approach was taken to visualize $A H$ gene expression. Slow increase upon wounding was recorded for $A H 1, A H 2, A H 3, A H 6$, and $A H 8$ transcripts, with maximal expression at later time points (Additional file 3: Figure S3). Because of rapid accumulation of JAs within the first hour after leaf wounding in Arabidopsis (Glauser et al. 2008; Heitz et al. 2012; Widemann et al. 2013), we speculated that genes whose expression was rapidly induced to high levels by wounding would be best candidates to participate in rice JA metabolism. AH7 combined a rapid and transient induction peaking at $0.5 \mathrm{hpw}$ and relatively high expression, whereas AH8 displayed the highest basal expression and moderate induction by $3 \mathrm{hpw}$.

We next addressed the transcriptional response of members in OsCYP94 or OsAH gene families, with focus on subclades predicted to encode JA-Ile metabolizing enzymes, in a parallel analysis of WT and jar1-1 mutant plants impaired in the major conjugating enzyme forming JA-Ile (Svyatyna et al. 2014). Most genes analyzed were induced by wounding to a lower extent but with similar kinetics in jar1-1 and in WT plants, as exemplified by JAZ11 gene used as a JA-Ile-regulated control (Fig. 2). This was particularly the case for OsCYP94B5, C2a, C4, and to a lesser extent for $D 7$ and $D 9$, indicating that their full induction depends on JA-Ile synthesis and signaling. In contrast, in jar1-1 OsAH7 was quantitatively similar to WT but peaked later ( $3 \mathrm{hpw}$ ), and OsAH8 expression was only marginally affected by jar1-1 mutation. These data identify CYP94B5, C2, C4 on the one hand, and $A H 7$ and $A H 8$ on the other hand as highly expressed and/or and wound-responsive encoding potential JA-Ile catabolizing enzymes in rice.

\section{Wounding Induces Strong, Partially JAR1-Dependent JA Accumulation and Catabolism in Rice}

Wounded plant material was then subjected to targeted JA profiling by UPLC-MS/MS. We quantified evolution of JAs upstream and downstream of bioactive JA-Ile, including OPDA, JA, JA-Ile and the catabolites 12OH-JA-Ile, $12 \mathrm{COOH}-\mathrm{JA}-\mathrm{Ile}$ and $12 \mathrm{OH}-\mathrm{JA}$, these latter deriving from JA-Ile-oxidizing and -cleaving activities. Resulting profiles are shown in Fig. 3. OPDA and JA accumulated upon wounding as expected, and were more abundant in jarl-1 than in WT, consistent with a block in JA-consuming JAR1 activity. JA-Ile abundance displayed a typical sharp increase peaking at $1 \mathrm{hpw}$ that readily declined afterwards (Heitz et al. 2012; Wakuta et al. 2011), and its wound-induced accumulation was efficiently abolished in jar1-1. Its oxidized derivative $12 \mathrm{OH}-J A-I l e$ paralleled JA-Ile profiles whereas second catabolite $12 \mathrm{COOH}-J \mathrm{~A}-$ Ile accumulated with more delayed kinetics. These results are in accordance with the concomitant induction of OsCYP94B5, C2a or C4 expression (Fig. 2) as putative JA-Ile oxidases. Furthermore, in response to wounding the abundance of oxidized conjugates was largely dependent on JAR1. Interestingly, 12OH-JA, which is known to be mostly generated by the sequential action of the CYP94-AH pathways in wounded Arabidopsis leaves (Smirnova et al. 2017; Widemann et al. 2013) (Additional file 1: Figure S1) is more abundant in jar1-1, suggesting a minor contribution of conjugated intermediates for its synthesis in wounded rice. Finally, JA-Phe, a minor conjugate reported in stressed Arabidopsis leaves (Kitaoka et al. 2014; Widemann et al. 2015) accumulated in trace amounts, that were more abundant in jar1-1 than in WT in wounded rice leaves. In contrast, no signal was detected for the following JA-amino-acid conjugates: JACys, JA-Gly, JA-Glu, JA-Pro, JA-Trp or JA-Tyr.

\section{Responses of JA-Ile Catabolic Genes to Salt Stress}

Because some impacts of the JA pathway on salt stress response have been reported (Hazman et al. 2015; Peethambaran et al. 2018), we examined the transcriptional behavior of the same set of selected CYP94 and $A H$ genes after seedling exposure to $100 \mathrm{mM} \mathrm{NaCl}$ stress. The material eventually developed symptoms of wilting as described in Hazman et al. (2015). Leaves were harvested 6, 24 or 72 $\mathrm{h}$ after onset of stress and extracted RNA was submitted to RT-qPCR analysis. CYP94B5 expression decreased to about 
-WT - jar1-1
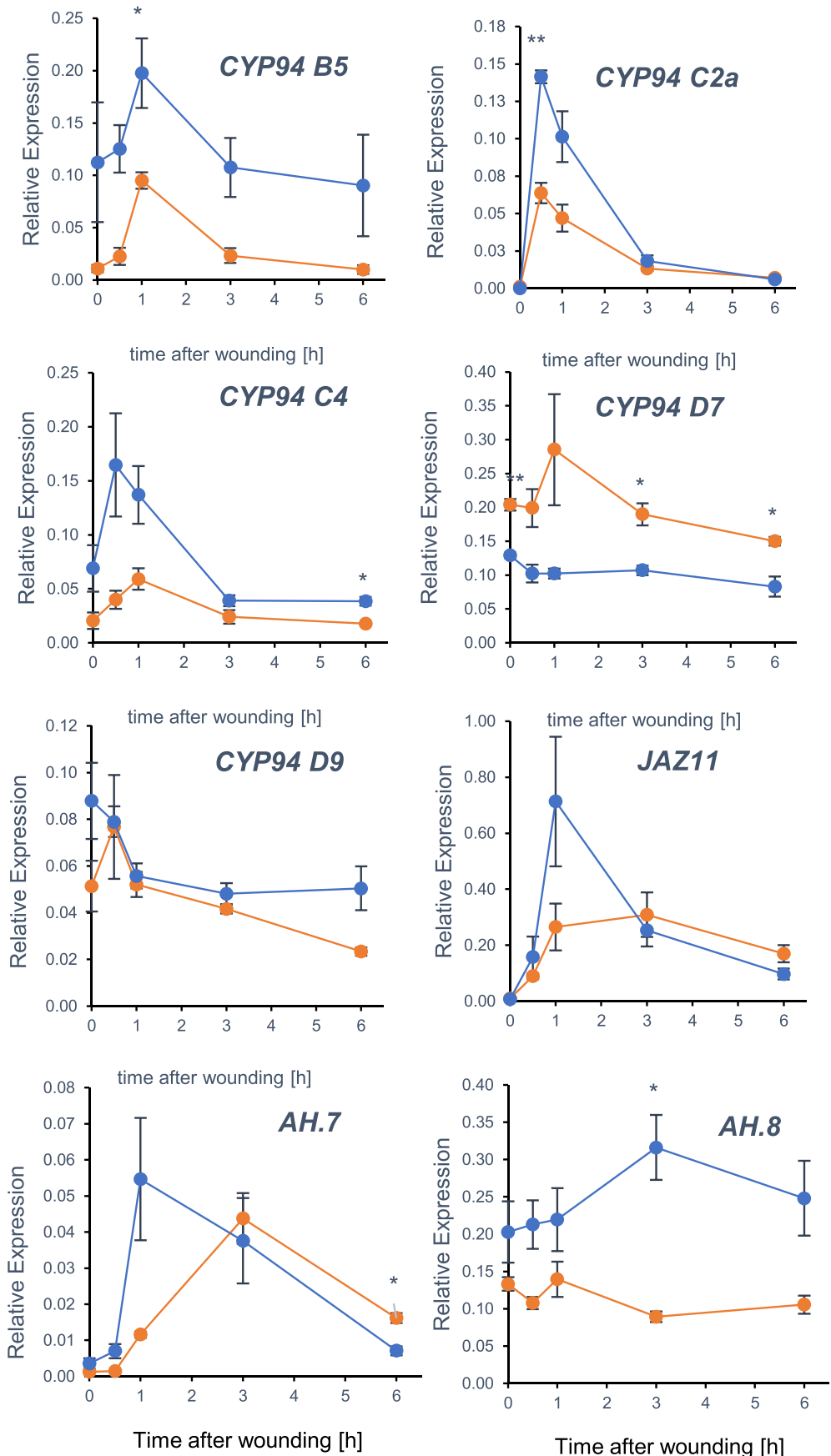

Fig. 2 Selected OsCYP94 and OsAH gene expression upon leaf wounding in WT and jar1-1 seedlings. Ten-day old (seedlings were submitted to leaf wounding and 2nd leaf was harvested at the indicated time points. Total RNA was submitted to RT-qPCR to reveal expression of indicated genes. Relative expression was determined using OsGAPDH as reference gene. Values are means and SD from 3 independent biological replicates

half control values upon salt exposure, independently of JAR1 (Fig. 4). CYP94C4 followed a similar trend in WT, but its expression was less affected in jar1-1. In contrast, CYP94C2a expression was strongly induced upon salt stress and this response was essentially maintained in jar11, similar to JAZ11 used as a JA-Ile-regulated marker.
CYP94D7 was down-regulated by salt in a JAR1independent manner, whereas CYP94D9 displayed a higher expression in jar1-1 control samples, that declined to WT levels in response to salt. Finally, $A H 8$ expression was unreactive to salt stress, with slightly higher expression in jar11. AH7 was found moderately upregulated by salt in WT, 

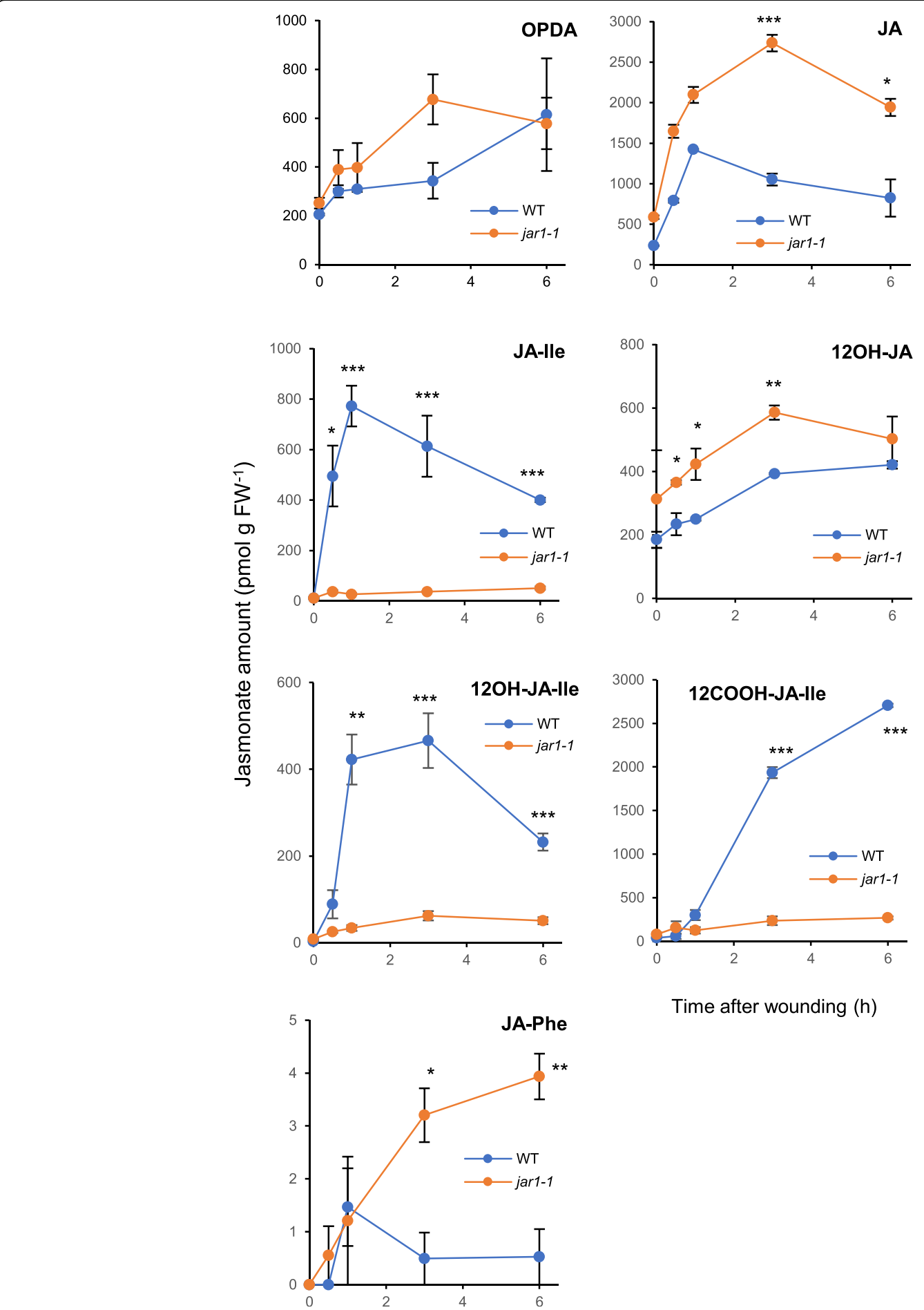

Time after wounding (h)

Fig. 3 Kinetic analysis of jasmonate accumulation in WT and jar1-1 plants upon mechanical leaf wounding. Ten-days old Nihonmasari WT and jar1-1 seedlings (same batch as in Fig. 2) were submitted to leaf wounding and 2nd leaf was harvested at the indicated time points. Leaves were extracted for jasmonate determination by UPLC-MS/MS. OPDA: 12-oxo-phytodienoic acid; JA: jasmonic acid; JA-lle: jasmonoyl-isoleucine; $12 \mathrm{OH}-J A$ : 12-hydroxy-jasmonic acid; 12OH-JA-lle: 12-hydroxy-jasmonoyl-isoleucine; 12COOH-JA-lle: 12-carboxy-jasmonoyl-isoleucine; JA-Phe: jasmonoylphenylalanine. Values are mean and SD of 3 independent biological replicates 

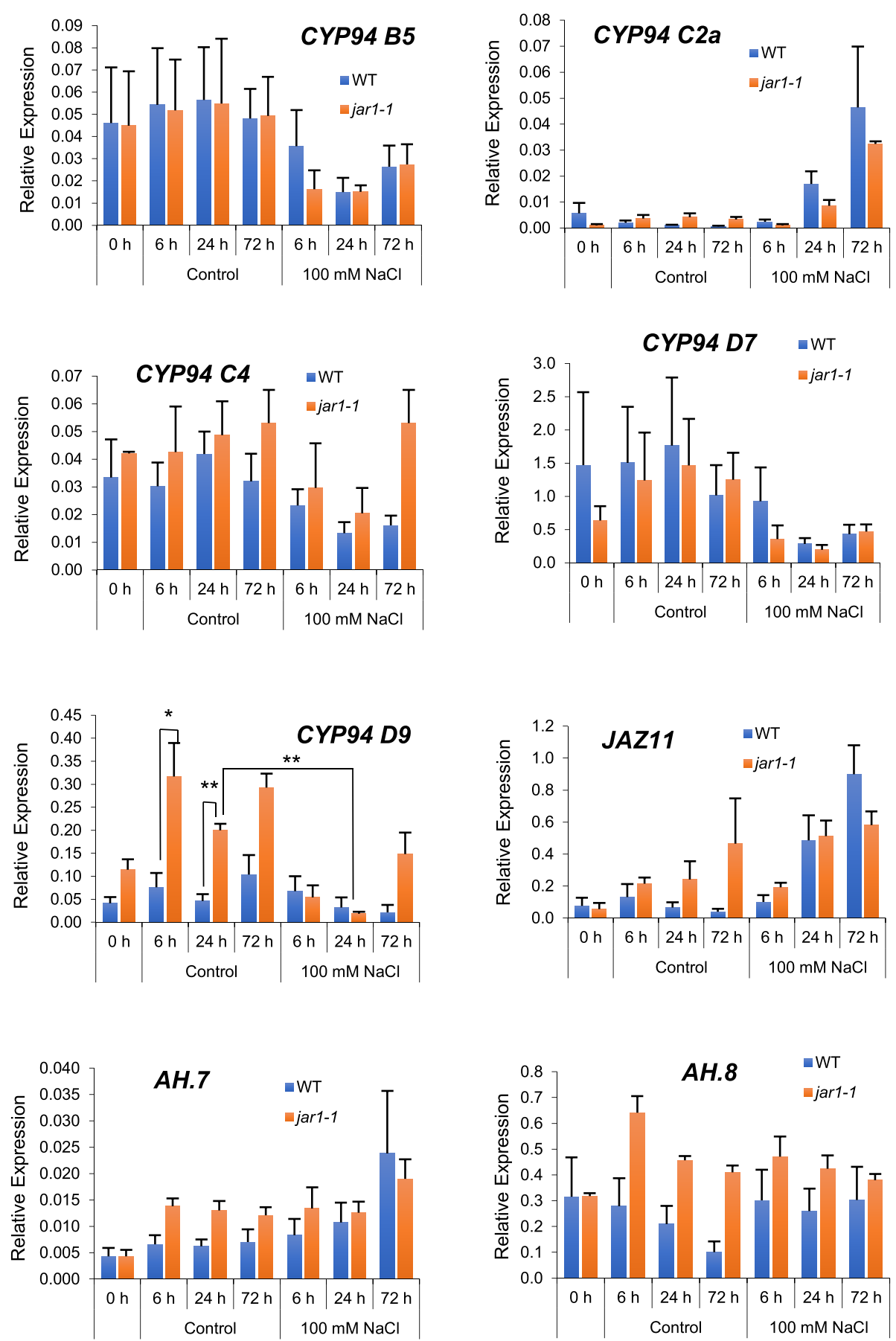

Fig. 4 Selected OsCYP94 and OsAH gene expression upon salt stress in WT and jar1-1 seedlings. Ten-days old seedlings were submitted to 100 $\mathrm{mM} \mathrm{NaCl}$ stress and 2nd leaf was harvested at the indicated time points. Total RNA was submitted to RT-qPCR to reveal expression of indicated genes. Relative expression was determined using OsUbiquitin 10 (OsUBQ10) and OsZCF61 as reference genes. Values are means and SD from 3 independent biological replicates

while its transcripts were slightly elevated in JAR1-deficient leaves. These data collectively show that salt stress induces specific changes in expression of CYP94 and $A H$ members and indicate a limited influence of JAR1-mediated signaling on these changes.

\section{Salt Stress Triggers JA Accumulation and Catabolism}

Leaves from salt-exposed and control seedlings were profiled for JAs content. All compounds analyzed increased steadily in response to stress in WT plants, with maximal levels at the latest time point recorded, after 
$72 \mathrm{~h}$ of exposure (Fig. 5). Like for wounding, OPDA and JA levels were enhanced by salt at most time points, but to higher levels in jar1-1 mutant compared to WT (Fig. 5). JA-Ile was very low in control plants but increased steadily in stressed WT material to about $1.4 \mathrm{nmolg}$ $\mathrm{FW}^{-1}$. Interestingly, lower but significant accumulation was also recorded in jar1-1. Accordingly, the JA-Ile catabolites $12 \mathrm{OH}-J \mathrm{~A}-\mathrm{Ile}$ and $12 \mathrm{COOH}-\mathrm{JA}$-Ile increased in response to salt exposure, with a JAR1-dependence which decreased along the pathway from JA-Ile to 12OH-JA-Ile and 12COOH-JA-Ile (Fig. 5). Because in jar1-1 JA-Ile still accumulated moderately, we examined transcript levels of the closely related homolog JAR2 that also conjugates JA to amino acids, including Ile (Svyatyna et al. 2014; Wakuta et al. 2011). As shown in Additional file 4: Figure S4A, JAR2 expression was found to be salt-repressed both in WT and in jar1-1 genotypes, making it unlikely that JAR2 contributes to salt-induced JA-Ile accumulation.

\section{Functional Analysis of CYP94 and AH Proteins}

In an attempt to determine JA-Ile-metabolizing capacities of encoded proteins, selected genes were expressed in heterologous hosts. To this end, CYP94B5, CYP94C2a and CYP94C4 cDNAs were cloned in pYeDP60 vector for expression in yeast. Microsome fractions were prepared as enzyme sources from yeast cells and incubated with synthetic JA-Ile before analyzing the formation of oxidation products by UPLC-MS/MS. As shown in Fig. 6a, microsomes from CYP94B5-expressing yeast yielded a clear signal matching characteristics of $12 \mathrm{OH}-$ JA-Ile standard compound, when incubation was performed in presence of NADPH cofactor. $12 \mathrm{COOH}-\mathrm{JA}$ Ile, the second oxidation product of AtCYP94C1 (Heitz

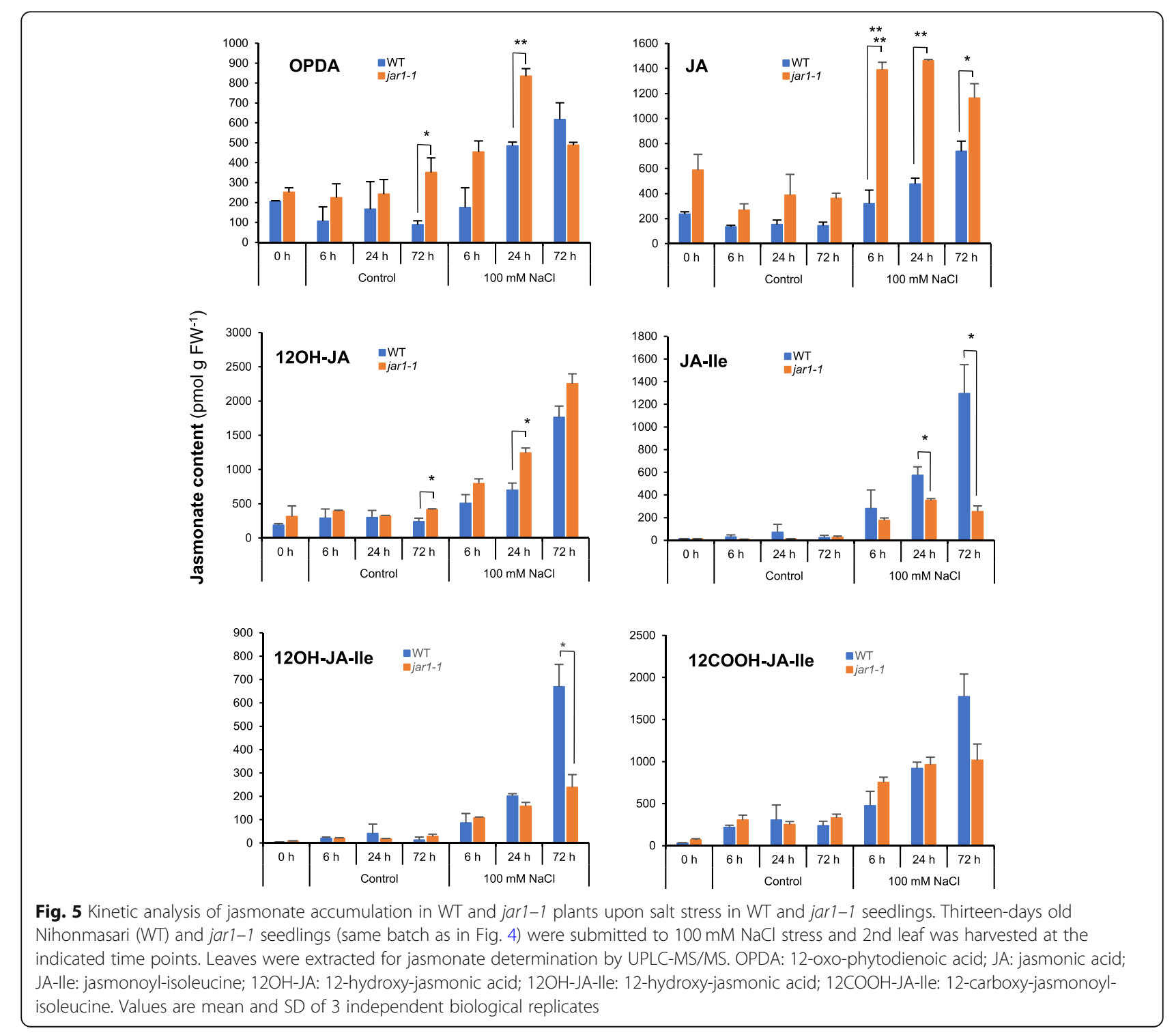




\section{a $\quad$ CYP94B5}
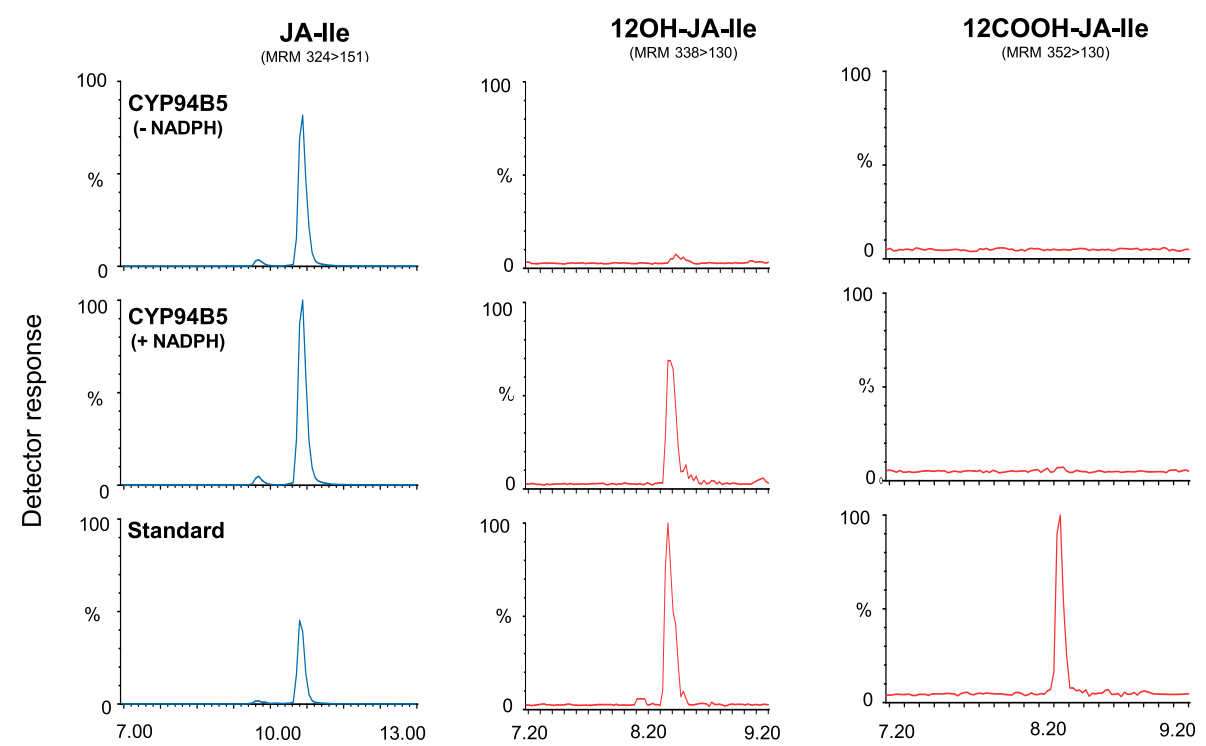

\section{b $\quad$ AH.8}
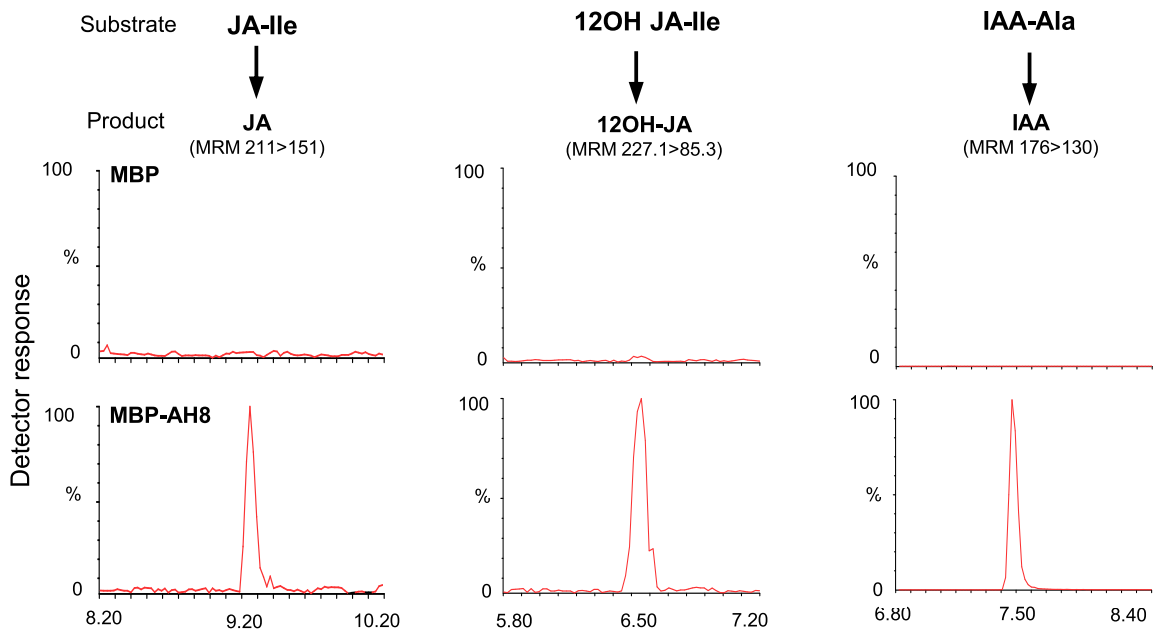

Fig. 6 In vitro enzymatic activity of recombinant OsCYP94B5 and OsAH8. a Microsomes of OsCYP94B5-expressing yeast were prepared and incubated for 30 min with $100 \mu \mathrm{M}$ JA-lle (blue traces) in absence or in presence of NADPH cofactor. Expected oxidation products (red traces) were identified based on the retention time and detection in multiple reaction monitoring mode of authentic $12 \mathrm{OH}-\mathrm{JA}-\| \mathrm{le}$ and $12 \mathrm{COOH}-\mathrm{JA}-l l e$ standards. b Recombinant his-tagged OsAH8 fused to Maltose Binding Protein (MBP) was affinity purified and incubated with $30 \mu \mathrm{M}$ of either of the following amino-acid conjugates: JA-lle, $12 \mathrm{OH}$-JA-lle or IAA-Ala. Respective cleavage products were searched for by detection based on retention time and multiple reaction monitoring mode of authentic JA, 12OH-JA or IAA standards. Analysis were performed in triplicates and one representative trace is shown

et al. 2012) could not be detected in incubations. Experiments with microsomes of CYP94C2- or CYP94C4transformed yeasts were unsuccessful in evidencing in vitro enzyme activity.

AH7 and AH8 coding sequences were cloned in expression vector pHMGWA, and expression of double-tagged
6xHis-Maltose Binding Protein (MBP)-AH was induced in bacterial cells. Fusion proteins of the expected sizes (HisMBP-AH8: $86.4 \mathrm{kDa}$; His-MBP-AH7: $92.7 \mathrm{kDa}$ ) could be isolated. His-MBP-AH8 was produced abundantly in soluble form and was purified by dextrin affinity chromatography followed by gel-filtration (Additional file 5: Figure 
S5a). His-MBP-AH7 expressed at lower abundance and was captured in bacterial lysates by immobilized metal affinity chromatography (IMAC) (Additional file 5: Figure S5b). Both proteins were next incubated with hormoneamino acid conjugates, typically JA- or auxin-amino acid conjugates, that were previously described as in vivo substrates of this class of enzymes (Rampey et al. 2004; Widemann et al. 2013; Zhang et al. 2016). Recombinant OsAH7 and OsAH8 were incubated with either JA-Ile, or with its oxidized derivative $12 \mathrm{OH}-J \mathrm{~A}-\mathrm{Il}$, or with the auxin conjugate IAA-Ala. Corresponding unconjugated hormonal compounds were searched for as evidence of cleaving activity. No activity could be recorded for OsAH7 with either substrate (not shown). In contrast, chromatograms shown in Fig. 6b illustrate that OsAH8, similarly to its Arabidopsis homolog AtIAR3, is able to generate free JA, $12 \mathrm{OH}-\mathrm{JA}$ and IAA from their respective conjugated substrates.

\section{Discussion}

Jasmonate signaling is governing major aspects of plant development and adaptation to environmental stress. Recent reports have revealed positive and negative contributions of JA signaling to biotic and abiotic threats (Hazman et al. 2015; Peethambaran et al. 2018; Riemann et al. 2013, 2015; $\mathrm{Wu}$ et al. 2015), calling for a better understanding of hormonal regulation. Catabolic processes affecting bioactive JA-Ile hormone homeostasis have been elucidated in Arabidopsis and shown to attenuate JA signaling (Heitz et al. 2012; Koo et al. 2011; Koo and Howe 2012; Widemann et al. 2013; Zhang et al. 2016). The precise actors and mechanisms of JA-Ile turnover were unknown in rice, but conservation of the oxidative pathway in this species was suggested by the correlation of OsCYP94C2b overexpression with higher in planta JA-Ile oxidation, leading to increased salt tolerance (Kurotani et al. 2015a, 2015b). Here we explored the rice CYP94 and $A H$ gene families, in a search for orthologs of the Arabidopsis JA-Ile oxidation and deconjugation enzymes (Heitz et al. 2016; Koo and Howe 2012). Similarity search readily identified large gene families; strikingly, subclades $B$ and $C$ of CYP94 encoding suspected JA-Ile oxidases only represented a minor portion of the OsCYP94 gene family, in contrast to subclade $D$ of unknown activity that displays up to 10 members. The additional occurrence of 3 subclade $E$ genes illustrates the high diversification of CYP94 genes/proteins in rice and questions the existence of distinct enzymatic activities. In contrast, the $A H$ gene family, with 9 members in rice had a comparable complexity to the 7-member Arabidopsis family.

To identify stress-responsive members, we examined transcriptional behavior of a subset of OsCYP94 and all $O s A H$ genes, in response to leaf wounding or salt exposure, two stresses known to cause rapid/massive, or slower changes in JA metabolism, respectively. Only members of the $C Y P 94 B$ and $C$ subclades were found to display a stereotypical expression peak at $0.5-1 \mathrm{hpw}$ that parallels the transient accumulation of JA-Ile, their presumed substrate, upon wounding (Heitz et al. 2012; Wakuta et al. 2011). Despite of sharing this profile, CYP94B4 and CYP94C3 have very low expression and likely are not major players of JA-Ile oxidation in wounded tissues. In contrast, OsCYP94B5, C2a and C4 displayed much higher expression, and similarly to Arabidopsis characterized CYP94B1, $B 3$ and C1 genes (Heitz et al. 2012), their induction was partially dependent on JA-Ile biosynthesis, as evidenced in the jar1-1 mutant. Quantitative, kinetic JA profiling was performed to reveal the characteristics of JA/JA-Ile catabolism in rice. Main features reported in Arabidopsis were conserved, with slower accumulation of its two oxidized derivatives. Therefore, OsCYP94B5, C2a and C4 are the prime candidates for wound-induced JA-Ile turn-over in rice. All 3 Ile-conjugates were nearly absent in jarl-1, but the minor conjugate JA-Phe was more abundant in this mutant, indicating it is produced by another conjugating enzyme, possibly boosted by the enhanced accumulation of JA precursor. JAR2, the closest JAR homolog in the GH3 family of rice, is unable to form JA-Phe in vitro (Svyatyna et al. 2014), and we established that its transcript levels decreased upon wounding in our experiments (Additional file 4: Figure S4B). Therefore, an unknown enzyme, possibly in the GH3 family may be involved in JA-Phe formation (Wakuta et al. 2011). In the $A H$ family, OsAH7 and OsAH8 behaved also similarly to their respective Arabidopsis AtILL6 and AtIAR3 closest homologs (Widemann et al. 2013), with a stable but high expression for OsAH8, and a dynamic pulse with weaker expression for OsAH7. These features do not allow to draw firm conclusions as to which AH contributes most to JA-Ile cleavage.

Transcriptional responses to salt exposure were contrasted. Only OsCYP94C2a was induced, similarly to OsJAZ11, and accompanied the increase in JA-Ile and its catabolites, whereas OsCYP94B5 and OsCYP94C4 were essentially down-regulated, as well as OsCYP94D7 and $D 9$. Therefore, OsCYP94C2a is the most likely actor of JA-Ile oxidation upon salt-stress, lending support to earlier data by Kurotani et al. (2015a, 2015b), who identified in a genetic screen the related $O s C Y P 94 C 2 b$ as a major single-gene contributor to salt tolerance. OsCYP94D7 and D9 decrease could underlie the need to shutdown a distinct enzyme activity upon salt response. OsAH7 and $O s A H 8$ transcripts only fluctuated marginally. As upon wounding, JA was hyperaccumulated in jar1-1 in salt-reacting leaves, indicating reduced JA metabolization, but Ile-conjugates were less reduced in this latter material, suggesting the contribution of another conjugating enzyme than JAR1. JAR2, the closest candidate to fulfill this function, is able to form JA-Ile in vitro (Svyatyna et al. 2014), but its contribution to saltinduced JA-Ile accumulation is not supported by the 
down-regulation of its transcripts. Currently, we are lacking information on alternative enzyme activities in the GH3 families, hence, more efforts are needed to clarify JAR1-independent JA conjugation. The fact that oxidized derivatives are less affected by jarl-1 mutation than JA-Ile may indicate that they undergo less deconjugation in this background and are therefore more stable.

Lastly, we set out to characterize directly enzyme activities of recombinant proteins. Absence of activity in OsCYP94C2a and C4 in yeast microsomes may be attributed to inactive conformation and/or insufficient expression. When carbon monoxide spectra of microsomes obtained from yeast transformed with OsCYP94C2a and C4 were recorded, a peak at about $420 \mathrm{~nm}$ rather than $450 \mathrm{~nm}$ was observed, suggesting the presence of misfolded heme-containing proteins (Luthra et al. 2011).

Our failure to assay recombinant OsCYP94C2a enzyme activity, combined with the absence of information on stress-induced expression of the closely related $\mathrm{OsCY}$ $P 94 C 2 b$ gene (Kurotani et al. 2015a) limits the knowledge of the relative contribution of these two isoforms to JA-Ile turnover. Affinity-purified OsAH7 also proved inactive, possibly due to mis-folding or hindrance by the MBP fusion. This is reminiscent of AtILL6, its Arabidopsis counterpart that is also notoriously difficult to express/assay (Widemann et al. 2013). However, for two enzymes, we could demonstrate JA-Ile-metabolizing activity. OsCY P94B5 displays JA-Ile oxidase activity in vitro, consistent with its Arabidopsis CYP94B orthologs (Heitz et al. 2012; Kitaoka et al. 2011; Koo et al. 2014). Rendering CYP94B5 expression salt-responsive for enhanced JA-Ile oxidative inactivation could be an attractive strategy to engineer salttolerance in rice. OsAH8 could be obtained in the active form, and its ability to cleave JA-aa and IAA-aa conjugates was shared with the bi-functional Arabidopsis IAR3 (Widemann et al. 2013; Zhang et al. 2016).

\section{Conclusions}

Our work sheds light on rice CYP94 and $A H$ gene families, in a context of hormone conjugate metabolism. CYP94 is so far limited to jasmonate substrates while some $\mathrm{AH}$ also readily catalyze cleavage of auxin conjugates (Zhang et al. 2016). The present evidence suggests that the JA-Ile catabolic network architecture is conserved in rice, both in terms of phylogeny and catalytic capacities: the closest homologous proteins display similar activities to enzymes characterized in Arabidopsis, but some unexpected regulations were also recorded. These findings and tools pave the way for stress-specific manipulation of JA-Ile catabolism for optimized output of the JA signaling pathway under various stresses.

\section{Materials and Methods}

\section{Phylogenetic Analysis}

Dr. D. Nelson (University of Tennesse, USA) kindly provided the names of CYP94 isoforms. The full length amino acid of rice cytochrome P450 (CYP94) and amidohydrolase $(\mathrm{AH})$ sequences were extracted from rice databases MSU (http://rice.plantbiology.msu.edu) and RAP (http:// rapdb.dna.affrc.go.jp) via blasting CYP94 and $\mathrm{AH}$ Arabidopsis protein sequences. CYP94 sequences were matched with sequences provided by D. Nelson. All sequences were then manually curated and loci identified in rice genome databases. A total of deduced 26 CYP94 and 16 AH fulllength amino acid sequences were analyzed using the phylogeny suite (Dereeper et al. 2008) and DENDROSCOPE v3 (Huson and Scornavacca 2012).

\section{Plant Materials, Growth and Stress Conditions}

In this study, Oryza sativa L. ssp. japonica cv. Nipponbare was used as the wild type, and jar1-1 (Riemann et al. 2008) was used as mutant. The caryopses were dehusked and surface sterilized according to (Hazman et al. 2015) and sowed on $0.4 \%$ phytoagar containing 5\% MS salts (Duchefa, Haarlem, The Netherlands) and incubated for 7 or 10 days in a culture room under continuous light at $25^{\circ} \mathrm{C}$. For wounding stress, 10 -day old seedlings were subjected to manual wounding using either metal forceps (Additional file 2: Figure S2, Additional file 3: Figure S3) or scissors (Fig. 2 and Fig. 3) to produce consistent wounds along the second leaf ( 5 and 7 wounds/ leaf, respectively). Wounded leaves were harvested kinetically $(0,0.5,1,3$ and $6 \mathrm{~h}$ after wounding) in 3 replicates and then stored at $80^{\circ} \mathrm{C}$ for quantitative gene expression analysis and jasmonate profiling. For salt stress, plant cultivation and treatments were accomplished according to Hazman et al. (2015). Briefly, surface sterilized caryopses of both WT and jar1-1 mutants were pre-germinated on $0.4 \%$ phytoagar for 7 days under continuous light of $120 \mu \mathrm{mol} \mathrm{m}^{-2} \mathrm{~s}^{-1}$ at $25^{\circ} \mathrm{C}$. The seedlings were transferred to microtubes with cut ends that were inserted on floating racks placed to a cylindric glass container containing 5\% MS medium as nutrient solution for extra 3 days. Subsequently, the solution was replaced by the same solution (control time course) or the same solution containing $\mathrm{NaCl}(100 \mathrm{mM})$. Leaves of both control and stressed plants were harvested kinetically $(0,6,24$ and $72 \mathrm{~h}$ after transferring into final solution) in multiple replicates and then stored at $-80^{\circ} \mathrm{C}$ for profiling of gene expression or jasmonate content.

\section{Total RNA Extraction and Quantitative Real-Time PCR}

For data shown in Additional file 2: Figure S2, Additional file 3: Figure S3, total RNA was isolated from the shoots of control, wounded and salt treated plants using Trizol Reagent (Molecular Research Center, Cincinatti, USA) according to the manufacturer's instructions. The 
cDNA synthesis was performed with cDNA synthesis kit (Invitrogen, Carlsbad, USA) using $1 \mu \mathrm{g}$ total RNA as a template. RT-qPCR (Reverse Transcriptase - quantitative PCR) was performed on $10 \mathrm{ng}$ cDNA with a SYBR green dye protocol using LightCycler 480 II instrument (Roche Applied Science, Penzberg, Germany) as follows: $95^{\circ} \mathrm{C}$ for $3 \mathrm{~min}$, and 40 cycles $\left(95^{\circ} \mathrm{C}\right.$ for $15 \mathrm{~s}$, annealing at $66^{\circ} \mathrm{C}$ for $30 \mathrm{~s}$ and extension at $72{ }^{\circ} \mathrm{C}$ for $30 \mathrm{~s}$ ). The gene expression levels in three biological replicates were calculated using the $\Delta \Delta^{\mathrm{Ct}}$ method.

For data shown in Fig. 2 and Fig. 4, RNA-extraction was performed using the innuPREP Plant RNA KIT (Analytik Jena AG, Jena, Germany) according to instructions provided by the manufacturer. The RNA was transcribed via a first strand cDNA using MuLV reverse transcriptase (New England Biolabs, Frankfurt, Germany) and oligo dT-primers in a two-step reaction. Quantitative analysis was performed on a CFX Touch real-time PCR system (Bio-Rad, Munich, Germany) according to the protocol of Svyatyna et al. (2014). The primer sequences for the genes of interest and reference genes are listed in Additional file 6: Table S1.

\section{Recombinant Heterologous Expression of OsCYP94B5 in Yeast and OsAH8 in Bacteria}

Coding sequence of OsCYP94B5, OsCYP94B4 and OsCY $P 94 C 2 a$ was generated by PCR amplification from rice genomic DNA cultivar Nipponbare. The forward and reverse primer sequences are given in Additional file 6: Table S1. The amplified PCR product was initially cloned in pGEM$\mathrm{T}$ easy vector according to manufacturer instructions (Promega) and then sequenced to verify error-free insert that was cloned in the BamH1 and EcoR1 sites of the yeast expression vector pYeDP60. OsCYP94B5, OsCYP94B4 and OsCYP94C2a proteins were produced in yeast optimized heterologous system as described in Heitz et al. (2012). P450 expression and quality control was performed by differential spectrophotometry as described in Gavira et al. (2013).

For heterologous expression of OsAH7 and OsAH8, open reading frame sequence deleted of the 69 (AH7) or 78 (AH8) N-terminal signal peptide-encoding nucleotides was amplified using a mixture (8:1) of hotstart Taq Polymerase and Phusion Taq Polymerase (Thermo Scientific, Illkirch-Graffenstaden, France) prior to the cloning in the pDONOR-Zeo vector (Thermo Scientific) in the DH5 $\alpha$ Escherichia coli strain. Inserts with error-free sequences were recombined into the expression vector pHMGWA. Plasmid was further transformed into the E. coli Rosetta 2 (DE3) strain (Merck, Darmstadt, Germany). Ice-cold bacterial pellets from isopropyl $ß-\mathrm{D}$-1-thiogalactopyranosideinduced $(0.5 \mathrm{mM})$ cultures were collected and kept frozen until use. For AH7 purification, pellet was thawed on ice for $30 \mathrm{~min}$ while resuspending in lysis buffer $(50 \mathrm{mM}$
Tris- $\mathrm{HCl} \mathrm{pH}$ 7.5, $300 \mathrm{mM} \mathrm{NaCl}, 20 \mathrm{mM}$ imidazole, $3 \mathrm{mg} /$ ml lysozyme) to an $\mathrm{OD}_{600}$ of 20 units. Bacteria were lysed by pulsed-sonication on ice for $4 \mathrm{~min}$. Lysate was clarified by centrifugation, supernatant was filtered and diluted with 1 vol buffer (Tris- $\mathrm{HCl} 50 \mathrm{mM}$ pH 8, NaCl $300 \mathrm{mM}$, glycerol 5\%) before loading on a His-Trap FF (1 ml capacity) column mounted on an Äkta purifier system (GE Healthcare, Velizy, France) and equilibrated with the same buffer. His-tagged protein was eluted with equilibration buffer containing $500 \mathrm{mM}$ imidazole. For AH8 purification, bacterial pellet was resuspended in $1 \mathrm{x}$ Phosphate Buffered Saline (PBS) pH 7.3 buffer to an $\mathrm{OD}_{600}$ of 20 units before lysis by pulsed sonication on ice for $4 \mathrm{~min}$. Clarified lysate was loaded on a MBP-Trap ( $1 \mathrm{ml}$ capacity) column pre-equilibrated with PBS solution and protein was eluted with equilibration buffer complemented with $10 \mathrm{mM}$ maltose. After analysis by SDS-PAGE, selected fractions were concentrated to $500 \mu \mathrm{L}$ and loaded on a Superdex 200 10/300 column equilibrated in 1x PBS pH 7.3. Concentration of proteins of interest was estimated via quantification with Bradford reagent using a bovine serum albumin calibration series.

\section{Enzyme Assays and Analysis}

For CYP94 assay, microsome incubations were performed as described in Widemann et al. (2015) with the following modifications: JA-Ile substrate concentration was $50 \mu \mathrm{M}$ and the reaction was stopped with $150 \mu \mathrm{L}$ methanol containing $0.2 \%$ acetic acid. The assays were centrifuged at $10000 \mathrm{~g}$ and the supernatant was used for LC-MS/MS analysis.

Amidohydrolase assay was performed in $200 \mu \mathrm{L}$ as described in Widemann et al. (2013) using $10 \mu \mathrm{g}$ affinitypurified protein and $30 \mu \mathrm{M}$ substrates (JA-Ile, 12OH-JA-Ile or IAA). Both types of enzyme activities were analyzed by LC-MS as described in Widemann et al. (2015) on a Waters Quattro Premier XE (Waters, Mildorf, MA USA) instrument, using the following detection parameters: in negative mode: JA 209 > 59; 12OH-JA $225>59$; 12OH-JAIle 338 > 130; 12COOH-JA-Ile $352>130$; in positive mode: JA-Ile 324 > 151; IAA-Ala 247 > 130; IAA $176>130$.

\section{Jasmonate Profiling}

Jasmonate profiling of rice leaves was performed as follows: about $50-100 \mathrm{mg}$ frozen plant material was extracted with 8 volumes of ice-cold extraction solution (MeOH:water:acetic acid 70:29:0.5) containing 9,10-dihydro-JA and 9,10-dihydro-JA-Ile as internal standards for workup recovery. Grinding was performed with a glassbead Precellys tissue homogenizer (Bertin Instruments, Montigny-le-Bretonneux, France) in $2 \mathrm{~mL}$ screw-capped tubes. After $30 \mathrm{~min}$ incubation at $4{ }^{\circ} \mathrm{C}$ on a rotating wheel, homogenates were cleared before concentration under a stream of $\mathrm{N}_{2}$ and overnight conservation at $-20^{\circ} \mathrm{C}$. After 
a second centrifuge step, extracts were submitted to LCMS/MS analysis on an EvoQ Elite LC-TQ (Bruker, Palaiseau, France). Column and chromatographic conditions were as described in Smirnova et al. (2017). Absolute quantifications were achieved by comparison of sample signals with dose-response curves established with pure compounds. Compound specific detections were performed in Multiple Reaction Monitoring Mode (MRM) using transitions described in Additional file 7: Table S2.

\section{Statistical Analysis}

All statistical analysis were performed using InfoStat 2015d (http:// www.infostat.com.ar). Comparisons of sample means were per- formed by one-way analysis of variance $(P<0.05$ or $P<0.01)$ and Tukey's post-hoc multiple comparisons tests $(P<0.05$ or $P<0.01)$, and significant differences of means were determined.

\section{Additional files}

Additional file 1: Figure S1. Arabidopsis simplified jasmonate metabolic pathway. JA-Ile oxidative and cleavage pathways are shown in yellow and blue backgrounds, respectively. (DOCX $48 \mathrm{~kb}$ )

Additional file 2: Figure S2. OsCYP94 gene expression upon leaf wounding. Ten-days old seedlings were submitted to leaf wounding and 2nd leaf was harvested at the indicated time points. Total RNA was submitted to RT-qPCR with specific primers for selected OsCYP94 genes (as indicated in Fig. 1). Relative expression was determined using Actin as reference gene. Values are means and SD from 3 replicate determinations. (DOCX $72 \mathrm{~kb}$ )

Additional file 3: Figure S3. OsAH gene expression upon leaf wounding. Ten-days old seedlings were submitted to leaf wounding and 2nd leaf was harvested at the indicated time points. Total RNA was submitted to RT-qPCR with $A H$-specific primers. Relative expression was determined using Actin as reference gene. Values are means and SD from 3 replicate determinations. (DOCX $57 \mathrm{~kb}$ )

Additional file 4: Figure S4. Expression profile of OsJAR2 upon salinity stress and leaf wounding in WT and jar1-1 seedlings. A: Ten-days old seedlings were submitted to leaf wounding and 2 nd leaf was harvested at the indicated time points. Total RNA was submitted to RT-qPCR with JAR2-specific primers. B: Ten-days old seedlings were submitted to 100 $\mathrm{mM} \mathrm{NaCl}$ stress and 2 nd leaf was harvested at the indicated time points. Total RNA was submitted to RT-qPCR to reveal JAR2-specific expression. Values are means and SD from 3 independent biological replicates. (DOCX $48 \mathrm{~kb}$ )

Additional file 5: Figure S5. SDS-PAGE analysis of recombinant AH8 and $\mathrm{AH} 7$ protein purification. $\mathrm{AH} 8$ and $\mathrm{AH} 7$ coding sequences were cloned in PHMGWA to be expressed as His-MBP-AH fusion proteins. a: Soluble AH8 bacterial lysate was submitted successively to amylose affinity (MBP-trap) followed by gel filtration chromatography. $\mathbf{b}$ : soluble AH7 bacterial lysate was submitted to immobilized metal-affinity chromatography (His-Trap). (DOCX 97 kb)

Additional file 6: Table S1. Sequences of primers for genes of interest used in the study. (DOCX $89 \mathrm{~kb}$ )

Additional file $\mathbf{7}$ Table S2. LC-MS parameters for endogenous jasmonate detection. (DOCX $38 \mathrm{~kb}$ )

\section{Acknowledgements}

We thank David Nelson (University of Tennessee, USA) for providing CYP94 sequences used for phylogeny. We are grateful to Pauline Delcros for help in OsAH7 expression and analysis.

\section{Authors' Contributions}

$\mathrm{MH}, \mathrm{MS}$ and SS performed plant treatment and analysis. LH purified recombinant protein, $\mathrm{AL}$ and $\mathrm{MH}$ produced yeast microsomes. $\mathrm{MH}, \mathrm{MS}$ and VM performed enzyme assays. JZ performed LC-MS analysis. FB and LM performed chemical synthesis of JAs. TH and MR designed research and wrote the paper. All authors read and approved the final manuscript.

\section{Funding}

$\mathrm{MH}$ was supported by a joint post-doctoral fellowship from the Institut Français d'Egypte (IFE, Cairo, Egypt) and the Science and Technology Development Fund (STDF, Egypt). MS was supported by the Erasmus exchange program of the European Union. VM and FB were recipients of a predoctoral fellowship from the Université de Strasbourg and the Ministère de l'Enseignement Supérieur et de la Recherche. Exchanges between KIT and IBMP were supported by Campus France Procope and German Academic Exchange Service (DAAD) funds. Publication of this article has been funded through the Open Access Publishing Fund of Karlsruhe Institute of Technology.

\section{Availability of Data and Materials}

All data supporting the conclusions of this article are provided within the article and its supplementary (Additional file 1: Figure S1, Additional file 2: Figure S2, Additional file 3: Figure S3; Additional file 6: Table S1, Additional file 7: Table S2).

\section{Ethics Approval and Consent to Participate}

Not applicable.

\section{Consent for Publication}

Not applicable.

\section{Competing Interests}

The authors declare that they have no competing interests.

\section{Author details}

${ }^{1}$ Institut de Biologie Moléculaire des Plantes (IBMP) du CNRS, Université de Strasbourg, Strasbourg, France. ${ }^{2}$ Agricultural Genetic Engineering Research Institute (AGERI), Agricultural Research Centre (ARC), Giza 12619, Egypt. ${ }^{3}$ Karlsruhe Institute of Technology, Botanical Institute, Karlsruhe, Germany. ${ }^{4}$ Synthèse Organique et Phytochimie (SOPhy), Institut de Chimie, Université de Strasbourg, CNRS, Strasbourg, France.

Received: 15 March 2019 Accepted: 5 June 2019

Published online: 25 June 2019

\section{References}

Aubert Y, Widemann E, Miesch L, Pinot F, Heitz T (2015) CYP94-mediated jasmonoyl-isoleucine hormone oxidation shapes jasmonate profiles and attenuates defence responses to Botrytis cinerea infection. J Exp Bot 66: 3879-3892

Cai Q, Yuan Z, Chen M, Yin C, Luo Z, Zhao X, Liang W, Hu J, Zhang D (2014) Jasmonic acid regulates spikelet development in rice. Nat Commun 5:3476

Campos ML, Kang JH, Howe GA (2014) Jasmonate-triggered plant immunity. J Chem Ecol 40:657-675

Chini A, Fonseca S, Fernandez G, Adie B, Chico JM, Lorenzo O, Garcia-Casado G, Lopez-Vidriero I, Lozano FM, Ponce MR, Micol JL, Solano R (2007) The JAZ family of repressors is the missing link in jasmonate signalling. Nature 448: 666-671

De Geyter N, Gholami A, Goormachtig S, Goossens A (2012) Transcriptional machineries in jasmonate-elicited plant secondary metabolism. Trends Plant Sci 17:349-359

Dereeper A, Guignon V, Blanc G, Audic S, Buffet S, Chevenet F, Dufayard JF, Guindon S, Lefort V, Lescot M, Claverie JM, Gascuel O (2008) Phylogeny.fr: robust phylogenetic analysis for the non-specialist. Nucleic Acids Res 36: W465-W469

Dhakarey R, Kodackattumannil Peethambaran P, Riemann M (2016) Functional analysis of Jasmonates in Rice through mutant approaches. Plants (Basel) 5(1) https://doi.org/10.3390/plants5010015

Du M, Zhao J, Tzeng DTW, Liu Y, Deng L, Yang T, Zhai Q, Wu F, Huang Z, Zhou M, Wang Q, Chen Q, Zhong S, Li CB, Li C (2017) MYC2 orchestrates a 
hierarchical transcriptional cascade that regulates jasmonate-mediated plant immunity in tomato. Plant Cell 29:1883-1906

Gavira C, Hofer R, Lesot A, Lambert F, Zucca J, Werck-Reichhart D (2013) Challenges and pitfalls of P450-dependent (+)-valencene bioconversion by Saccharomyces cerevisiae. Metab Eng 18:25-35

Glauser G, Grata E, Dubugnon L, Rudaz S, Farmer EE, Wolfender IL (2008) Spatial and temporal dynamics of jasmonate synthesis and accumulation in Arabidopsis in response to wounding. J Biol Chem 283:16400-16407

Hazman M, Hause B, Eiche E, Nick P, Riemann M (2015) Increased tolerance to salt stress in OPDA-deficient rice ALLENE OXIDE CYCLASE mutants is linked to an increased ROS-scavenging activity. J Exp Bot 66:3339-3352

Heitz T, Smirnova E, Widemann E, Aubert Y, Pinot F, Menard R (2016) The rise and fall of jasmonate biological activities. Subcell Biochem 86:405-426

Heitz T, Widemann E, Lugan R, Miesch L, Ullmann P, Desaubry L, Holder E, Grausem B, Kandel S, Miesch M, Werck-Reichhart D, Pinot F (2012) Cytochromes P450 CYP94C1 and CYP94B3 catalyze two successive oxidation steps of plant hormone Jasmonoyl-isoleucine for catabolic turnover. J Biol Chem 287:6296-6306

Hibara K, Isono M, Mimura M, Sentoku N, Kojima M, Sakakibara H, Kitomi Y, Yoshikawa T, Itoh J, Nagato Y (2016) Jasmonate regulates juvenile-to-adult phase transition in rice. Development 143:3407-3416

Hickman R, Van Verk MC, Van Dijken AJH, Mendes MP, Vroegop-Vos IA, Caarls L, Steenbergen M, Van der Nagel I, Wesselink GJ, Jironkin A, Talbot A, Rhodes J, De Vries M, Schuurink RC, Denby K, Pieterse CMJ, Van Wees SCM (2017) Architecture and dynamics of the jasmonic acid gene regulatory network. Plant Cell 29:2086-2105

Huson DH, Scornavacca C (2012) Dendroscope 3: an interactive tool for rooted phylogenetic trees and networks. Syst Biol 61:1061-1067

Kazan K (2015) Diverse roles of jasmonates and ethylene in abiotic stress tolerance. Trends Plant Sci 20:219-229

Kitaoka N, Kawaide H, Amano N, Matsubara T, Nabeta K, Takahashi K, Matsuura H (2014) CYP94B3 activity against jasmonic acid amino acid conjugates and the elucidation of 12-O-beta-glucopyranosyl-jasmonoyl-L-isoleucine as an additional metabolite. Phytochemistry 99:6-13

Kitaoka N, Matsubara T, Sato M, Takahashi K, Wakuta S, Kawaide H, Matsui H, Nabeta K, Matsuura H (2011) Arabidopsis CYP94B3 encodes jasmonyl-Lisoleucine 12-hydroxylase, a key enzyme in the oxidative catabolism of jasmonate. Plant Cell Physiol 52:1757-1765

Koo AJ (2018) Metabolism of the plant hormone jasmonate: a sentinel for tissue damage and master regulator of stress response. Phytochem Rev 17:51 https://doi.org/10.1007/s11101-017-9510-8:1-30

Koo AJ, Cooke TF, Howe GA (2011) Cytochrome P450 CYP94B3 mediates catabolism and inactivation of the plant hormone jasmonoyl-L-isoleucine. Proc Natl Acad Sci U S A 108:9298-9303

Koo AJ, Howe GA (2012) Catabolism and deactivation of the lipid-derived hormone jasmonoyl-isoleucine. Front Plant Sci 3:19

Koo AJ, Thireault C, Zemelis S, Poudel AN, Zhang T, Kitaoka N, Brandizzi F, Matsuura H, Howe GA (2014) Endoplasmic reticulum-associated inactivation of the hormone jasmonoyl-L-isoleucine by multiple members of the cytochrome P450 94 family in Arabidopsis. J Biol Chem 289:29728-29738

Kurotani K, Hayashi K, Hatanaka S, Toda Y, Ogawa D, Ichikawa H, Ishimaru Y, Tashita R, Suzuki T, Ueda M, Hattori T, Takeda S (2015a) Elevated levels of CYP94 family gene expression alleviate the jasmonate response and enhance salt tolerance in rice. Plant Cell Physiol 56:779-789

Kurotani K, Yamanaka K, Toda Y, Ogawa D, Tanaka M, Kozawa H, Nakamura H, Hakata M, Ichikawa H, Hattori T, Takeda S (2015b) Stress tolerance profiling of a collection of extant salt-tolerant rice varieties and transgenic plants overexpressing abiotic stress tolerance genes. Plant Cell Physiol 56:1867-1876

Liu L, Zou Z, Qian K, Xia C, He Y, Zeng H, Zhou X, Riemann M, Yin C (2017) Jasmonic acid deficiency leads to scattered floret opening time in cytoplasmic male sterile rice Zhenshan 97A. J Exp Bot 68:4613-4625

Liu Z, Zhang S, Sun N, Liu H, Zhao Y, Liang Y, Zhang L, Han Y (2015) Functional diversity of jasmonates in rice. Rice $(N$ Y) 8:42

Lu J, Robert CA, Riemann M, Cosme M, Mene-Saffrane L, Massana J, Stout MJ, Lou Y, Gershenzon J, Erb M (2015) Induced jasmonate signaling leads to contrasting effects on root damage and herbivore performance. Plant Physiol 167:1100-1116

Luthra A, Denisov IG, Sligar SG (2011) Spectroscopic features of cytochrome P450 reaction intermediates. Arch Biochem Biophys 507:26-35

Peethambaran PK, Glenz R, Honinger S, Shahinul Islam SM, Hummel S, Harter K, Kolukisaoglu U, Meynard D, Guiderdoni E, Nick P, Riemann M (2018) Salt- inducible expression of OsJAZ8 improves resilience against salt-stress. BMC Plant Biol 18:311

Rampey RA, LeClere S, Kowalczyk M, Ljung K, Sandberg G, Bartel B (2004) A family of auxin-conjugate hydrolases that contributes to free indole-3-acetic acid levels during Arabidopsis germination. Plant Physiol 135:978-988

Riemann M, Dhakarey R, Hazman M, Miro B, Kohli A, Nick P (2015) Exploring jasmonates in the hormonal network of drought and salinity responses. Front Plant Sci 6:1077

Riemann M, Haga K, Shimizu T, Okada K, Ando S, Mochizuki S, Nishizawa Y, Yamanouchi U, Nick P, Yano M, Minami E, Takano M, Yamane H, lino M (2013) Identification of rice Allene oxide cyclase mutants and the function of jasmonate for defence against Magnaporthe oryzae. Plant J 74:226-238

Riemann M, Riemann M, Takano M (2008) Rice JASMONATE RESISTANT 1 is involved in phytochrome and jasmonate signalling. Plant Cell Environ 31:783-792

Schaller A, Stintzi A (2009) Enzymes in jasmonate biosynthesis - structure, function, regulation. Phytochemistry 70:1532-1538

Shrivastava P, Kumar R (2015) Soil salinity: a serious environmental issue and plant growth promoting bacteria as one of the tools for its alleviation. Saudi J Biol Sci 22:123-131

Smirnova E, Marquis V, Poirier L, Aubert Y, Zumsteg J, Menard R, Miesch L, Heitz $T$ (2017) Jasmonic acid oxidase 2 hydroxylates jasmonic acid and represses basal defense and resistance responses against Botrytis cinerea infection. Mol Plant 10:1159-1173

Svyatyna K, Jikumaru Y, Brendel R, Reichelt M, Mithofer A, Takano M, Kamiya Y, Nick P, Riemann M (2014) Light induces jasmonate-isoleucine conjugation via OsJAR1-dependent and -independent pathways in rice. Plant Cell Environ 37:827-839

Thines B, Katsir L, Melotto M, Niu Y, Mandaokar A, Liu G, Nomura K, He SY, Howe GA, Browse J (2007) JAZ repressor proteins are targets of the SCF(COI1) complex during jasmonate signalling. Nature 448:661-665

Wakuta S, Suzuki E, Saburi W, Matsuura H, Nabeta K, Imai R, Matsui H (2011) OsJAR1 and OsJAR2 are jasmonyl-L-isoleucine synthases involved in woundand pathogen-induced jasmonic acid signalling. Biochem Biophys Res Commun 409:634-639

Wasternack C, Hause B (2013) Jasmonates: biosynthesis, perception, signal transduction and action in plant stress response, growth and development. An update to the 2007 review in annals of botany. Ann Bot 111:1021-1058

Widemann E, Grausem B, Renault H, Pineau E, Heinrich C, Lugan R, Ullmann P, Miesch L, Aubert Y, Miesch M, Heitz T, Pinot F (2015) Sequential oxidation of Jasmonoyl-phenylalanine and Jasmonoyl-isoleucine by multiple cytochrome P450 of the CYP94 family through newly identified aldehyde intermediates. Phytochemistry 117:388-399

Widemann E, Miesch L, Lugan R, Holder E, Heinrich C, Aubert Y, Miesch M, Pinot F, Heitz T (2013) The amidohydrolases IAR3 and ILL6 contribute to jasmonoyl-isoleucine hormone turnover and generate 12-hydroxyjasmonic acid upon wounding in Arabidopsis leaves. J Biol Chem 288:31701-31714

Woldemariam MG, Onkokesung N, Baldwin IT, Galis I (2012) Jasmonoyl-Lisoleucine hydrolase $1(\mathrm{JIH} 1)$ regulates jasmonoyl-L-isoleucine levels and attenuates plant defenses against herbivores. Plant J 72:758-767

Wu H, Ye H, Yao R, Zhang T, Xiong L (2015) OsJAZ9 acts as a transcriptional regulator in jasmonate signaling and modulates salt stress tolerance in rice. Plant Sci 232:1-12

Yamada S, Kano A, Tamaoki D, Miyamoto A, Shishido H, Miyoshi S, Taniguchi S, Akimitsu K, Gomi K (2012) Involvement of OsJAZ8 in jasmonate-induced resistance to bacterial blight in rice. Plant Cell Physiol 53:2060-2072

Ye M, Luo SM, Xie JF, Li YF, Xu T, Liu Y, Song YY, Zhu-Salzman K, Zeng RS (2012) Silencing COI1 in rice increases susceptibility to chewing insects and impairs inducible defense. PLoS One 7:e36214

Zhang T, Poudel AN, Jewell JB, Kitaoka N, Staswick P, Matsuura H, Koo AJ (2016) Hormone crosstalk in wound stress response: wound-inducible amidohydrolases can simultaneously regulate jasmonate and auxin homeostasis in Arabidopsis thaliana. J Exp Bot 67:2107-2120

\section{Publisher's Note}

Springer Nature remains neutral with regard to jurisdictional claims in published maps and institutional affiliations. 